\title{
Fatigue in patients with COPD participating in a pulmonary rehabilitation program
}

This article was published in the following Dove Press journal: International Journal of Chronic Obstructive Pulmonary Disease 10 September 2010

Number of times this article has been viewed

\author{
Cindy J Wong' \\ Donna Goodridge' \\ Darcy D Marciniuk ${ }^{2}$ \\ Donna Rennie ${ }^{1,3}$ \\ 'College of Nursing, ${ }^{2}$ College of \\ Medicine, ${ }^{3}$ Canadian Centre for \\ Health and Safety in Agriculture, \\ University of Saskatchewan, \\ Saskatoon, Canada
}

Correspondence: Donna Goodridge College of Nursing, University of Saskatchewan, Health Sciences Building, I07 Wiggins Road, Saskatoon, Saskatchewan, S7N 5E5, Canada

Tel + I 306966 |478

$\mathrm{Fax}+\mathrm{I} 3069666703$

Email donna.goodridge@usask.ca
Background: Fatigue is a distressing, complex, multidimensional sensation common in individuals with chronic obstructive pulmonary disease (COPD). While fatigue negatively impacts functional performance and quality of life, there has been little study of the fatigue that affects participants in pulmonary rehabilitation programs. The purpose of this study was to examine the emotional, behavioral, cognitive, and physical dimensions of fatigue and their relationships to dyspnea, mental health, sleep, and physiologic factors.

Patients and methods: A convenience sample of 42 pulmonary rehabilitation participants with COPD completed self-report questionnaires which measured dimensions of fatigue using the Multidimensional Fatigue Inventory, anxiety and depression using the Hospital Anxiety and Depression Scale, and sleep quality using the Pittsburgh Sleep Quality Index. Data on other clinical variables were abstracted from pulmonary rehabilitation program health records.

Results: Almost all (95.3\%) participants experienced high levels of physical fatigue. High levels of fatigue were also reported for the dimensions of reduced activity (88.1\%), reduced motivation $(83.3 \%)$, mental fatigue $(69.9 \%)$, and general fatigue $(54.5 \%)$. Close to half $(42.9 \%)$ of participants reported symptoms of anxiety, while almost one quarter $(21.4 \%)$ reported depressive symptoms. Age was related to the fatigue dimensions of reduced activity $(\rho=0.43, P<0.01)$ and reduced motivation $(\rho=0.31, P<0.05)$. Anxiety was related to reduced motivation $(\rho=-0.47$, $P<0.01$ ). Fatigue was not associated with symptoms of depression, sleep quality, gender, supplemental oxygen use, smoking status, or Medical Research Council dyspnea scores.

Conclusions: Fatigue (particularly the physical and reduced motivation dimensions of fatigue) was experienced by almost all participants with COPD attending this pulmonary rehabilitation program. Fatigue affected greater proportions of participants than either anxiety or depression. The high prevalence of fatigue may impact on enrolment, participation, and attrition in pulmonary rehabilitation programs. Further investigation of the nature, correlates, and impact of fatigue in this population is required.

Keywords: COPD, fatigue, pulmonary rehabilitation, anxiety, depression, sleep quality

\section{Introduction}

Chronic obstructive pulmonary disease (COPD) is a debilitating lung condition that manifests in shortness of breath, activity limitation, increased sputum production, and cough. ${ }^{1}$ Currently the fifth leading cause of death and disease burden globally, ${ }^{1}$ COPD is characterized by significant physical and psychosocial challenges. In contrast with a prevalence rate of $18.3 \%-25 \%$ in the general population, 2,3 fatigue is "almost always" experienced by $43 \%-58 \%$ of persons with COPD. ${ }^{4-6}$ Fatigue is one of the most distressing symptoms of this illness, ${ }^{7,8}$ and significantly impairs both functional performance and quality of life. ${ }^{9,10}$ The aim of this study was to examine fatigue in individuals with 
COPD enrolled in a pulmonary rehabilitation program from a multidimensional perspective and to assess the relationship of fatigue to anxiety, depression, sleep quality, dyspnea, and physiologic factors.

Individuals living with COPD in the community have a moderate to high prevalence of symptoms. ${ }^{6}$ The experience of unpleasant symptoms, such as fatigue, contributes to impaired quality of life. ${ }^{11}$ Fatigue imposes limitations on motivation, concentration, and the ability to engage in everyday activities such as work, household chores, and social pastimes, ${ }^{12}$ engendering frustration, depression, grief, and a sense of loss of control. ${ }^{13}$

Measurement of fatigue has been plagued by a lack of consensus on its definition, ${ }^{14,15}$ and a lack of agreement on its dimensions. ${ }^{16}$ For the purposes of this study, fatigue was defined as "a subjective, unpleasant symptom which incorporates total body feelings ranging from tiredness to exhaustion, creating an unrelenting overall condition which interferes with individuals' ability to function to their normal capacity". ${ }^{13}$ The Multidimensional Fatigue Inventory (MFI) was used in this study, ${ }^{17}$ and has been found to be valid and reliable in measuring fatigue in the chronically unwell population. ${ }^{17}$

Moderate to strong positive relationships have been reported between fatigue and mood states such as anxiety, irritability and depression, ${ }^{5,18}$ and sleep quality. ${ }^{14}$ In primary care populations, patients with fatigue receive a lifetime diagnosis of depression or anxiety more frequently than those without fatigue, ${ }^{19}$ and there appears to be a longitudinal relationship between the severity of fatigue and impaired functioning and psychologic symptoms. ${ }^{20}$ Poor quality of sleep is a common symptom among older adults, and has been attributed in large part to the presence of chronic illness in this population. ${ }^{21}$

While fatigue has been found to be related to dyspnea, ${ }^{22}$ it does not appear to be directly related to measures of pulmonary function. ${ }^{10}$ Pulmonary rehabilitation programs are designed to optimize physical and social performance and autonomy ${ }^{1}$ in persons with COPD. The efficacy of pulmonary rehabilitation programs in improving functional capacity and disease-specific health perception is well documented. ${ }^{23}$

Fatigue has not been a major focus of study to date in persons with COPD attending pulmonary rehabilitation programs. Benzo et al found that pulmonary rehabilitation resulted in improvements in energy fatigue scores on the SF-36. ${ }^{24}$ In contrast, a recent randomized controlled trial conducted by Theander et al was unable to detect improvements in fatigue levels following pulmonary rehabilitation. ${ }^{15}$
Inconsistent measurement of fatigue between studies may account for this discrepancy.

Limited research has been conducted to examine various factors that influence fatigue as well as some dimensions of fatigue in individuals with COPD. Our study examined four dimensions of subjective fatigue in a group of individuals with COPD and several factors previously identified in the literature as influencing fatigue.

\section{Methods}

\section{Research design}

A descriptive, cross-sectional survey design using previously validated measures was used to assess fatigue. The theory of unpleasant symptoms was used as the conceptual model guiding this study. ${ }^{25}$ Subjective fatigue, the unpleasant symptom under consideration, was conceptualized to include four dimensions: emotional, behavioral, cognitive, and physical. These four dimensions were explored in terms of their relationships to physiologic, psychologic, and situational factors which may influence fatigue.

\section{Setting and participants}

The setting was an outpatient pulmonary rehabilitation program offered at two sites in the city of Saskatoon in the Saskatoon Health Region. This program is available to patients with COPD confirmed by spirometry, ${ }^{1}$ and who are symptomatic regardless of severity of disease. ${ }^{22}$ Eligibility criteria for the study included a physician-confirmed diagnosis of COPD, able to read, write, understand, and communicate in English, and aged 18 years or older. The program is designed for a duration of 12 months; however, participants may continue to participate past that time. Participants engage in monitored physical activity and receive education on numerous topics. All participants completed an eight-week initiation program in pulmonary rehabilitation and were currently in the maintenance phase. Ongoing program evaluation reveals clinically meaningful and statistically significant improvements in exercise limitation (improved walking distance) and quality of life (reduced St George's Respiratory Questionnaire score) at 3, 6, and 12 months following enrolment (personal communication, D Marciniuk, Medical Director, Saskatoon Pulmonary Rehabilitation Program).

Recruitment and data collection took place simultaneously between October 2008 and December 2008, for a total period of two months. Participants who were determined by the program coordinator to have met the eligibility criteria for the study were approached by the researcher. Forty-two of the 45 participants approached agreed to participate, giving 
a participation rate of $93.3 \%$. The researcher met with each participant individually and distributed a demographic form, as well as several questionnaires which assessed fatigue, anxiety, depression, and sleep quality. Participants were asked to complete all forms during that meeting. The participants were deemed stable by their physician to engage in the physical exercise component of the program. Some of the participants had completed the 12-month program, and continued to enroll for maintenance purposes, while others had not completed the program. Ethical approval was obtained from the University of Saskatchewan Behavioral Research Ethics Board, and operational approval was obtained from the Saskatoon Health Region. All subjects provided informed consent to participate.

\section{Instruments}

\section{Fatigue}

The MFI-20 was used to measure subjective fatigue. ${ }^{17}$ The MFI-20 is comprised of 20 items divided into five subscales which measure the primary dimensions of fatigue, including general fatigue, physical fatigue, reduced activity, reduced motivation, and mental fatigue. The general and physical fatigue subscales measured the physical dimension of fatigue. The reduced activity, reduced motivation, and mental fatigue subscales measured the behavioral, emotional, and cognitive dimensions of fatigue, respectively. The five dimensions of fatigue in the instrument were based on how fatigue could be expressed, which included a general evaluation about one's functioning, physically feeling tired, cognitive indications, being less motivated, and engaging in fewer activities. ${ }^{26}$ Each of the five subscales relate to these dimensions and contain four items which are rated on a scale from 1 to 5 , where one indicates "yes, that is true" and five indicates "no, that is not true". A total score is calculated for each subscale, where scores range from 4 to 20 , and higher scores indicating more fatigue. The instrument demonstrates good internal consistency with average Cronbach's alpha scores of $0.86 .{ }^{26,27}$ The MFI has previously been used in studies of persons with COPD. ${ }^{28,29}$

\section{Anxiety and depression}

Anxiety and depression were measured using the Hospital Anxiety and Depression Scale (HADS). ${ }^{30}$ The ease, speed, patient acceptance, and validated psychometric properties of the HADS allow it to be applied to a wide variety of clinical populations where significant anxiety and depression may coexist with the manifestation of physical illness, including COPD. ${ }^{31}$ This widely used instrument contains 14 items, of which seven items measure symptoms of depression occurring in the past week, while the remainder measure symptoms of anxiety occurring in the past week. Each item is rated on a four-point scale; item scores are summed to yield subscores for anxiety and depression. Scores can range between 0 and 21 . Scores between 8 and 10 on either subscale suggest mild anxiety or depression, while scores between 11 and 14 are considered moderate, and those 15 or above are considered severe.

\section{Sleep quality}

The Pittsburgh Sleep Quality Index (PSQI) was used to measure sleep quality. ${ }^{32}$ The scale is comprised of 19 items divided into seven components: sleep quality, sleep latency, sleep duration, habitual sleep efficiency, sleep disturbances, use of sleep medications, and daytime dysfunction. Each item is rated on a scale from 0 to 3 , where zero indicates no difficulty and three indicates severe difficulty. A Global Sleep Quality Index is calculated with scores ranging between 0 and 21; scores of 5 or more on the index indicate poor sleep quality. ${ }^{32}$ The PSQI has been found to have high internal consistency, ${ }^{32,33}$ test-retest reliability, concurrent validity, and construct validity. ${ }^{32}$

\section{Dyspnea}

The Medical Research Council (MRC) dyspnea scale was used to assess dyspnea. ${ }^{34}$

\section{Activity limitation}

The most recent 6-minute walk test (6MWT) available in the health record was used as a measure of functional activity limitation. ${ }^{35}$ The $6 \mathrm{MWT}$ is performed at the start of the program, as well as after three, six, and 12 months of participation in the program.

\section{Additional data}

Further data gathered from the health record included smoking status, duration of enrolment in pulmonary rehabilitation, comorbidities, supplemental oxygen use, respiratory medications, and the most current oxygen saturation readings from pulse oximetry (recorded immediately after walking).

\section{Data analysis}

Data were entered into SPSS software (v. 16.0; SPSS Inc., Chicago, IL) and recoded as required according to the questionnaires' scoring instructions. Descriptive and Spearman's rho correlational analyses were conducted. Comparisons in proportions between groups were completed using Chi-square or 
Kruskal-Wallis tests of proportion. Statistical significance for the bivariate analysis was set at $P \leq 0.05$.

\section{Results}

Forty-two of the 45 participants enrolled in the study, and $40 \%$ (17) were male, with a mean age of 72.9 years (standard deviation $[\mathrm{SD}] \pm 6.5$, range 56-87). Although recent spirometry results from only 14 participants were available in the program site health records, they indicate that those subjects had a forced expiratory volume in one second/ forced vital capacity $\left(\mathrm{FEV}_{1} / \mathrm{FVC}\right)$ of less than 0.7 , and the mean $\mathrm{FEV}_{1}$ was $47.8 \%$ of the predicted, confirming some presence of severe COPD in this limited sample. ${ }^{1}$ Only 14 participants had recent spirometry results kept on file at their pulmonary rehabilitation facility because the results are normally kept at their physicians' office and are sent to the pulmonary rehabilitation facility upon request. Due to the limited number of spirometry results available, we did not find a correlation between severity of COPD and fatigue. Data on the total number of comorbidities were gathered, which included any coexisting diseases and excluded the diagnosis of COPD. The median number was three, and $52.4 \%$ (22) had three or more comorbidities. More than three-quarters of participants (33) were exsmokers, $4.8 \%$ (2) were nonsmokers, and 16.7\% (7) were current smokers. Supplemental oxygen was used by $26.2 \%$ (11) of the participants. There were a total of 16 participants who began pulmonary rehabilitation between 2002 and 2006, seven who began in 2007, and 19 who began in 2008. Most participants had attended pulmonary rehabilitation for just over two years. The average length of attendance was 25 months ( $\mathrm{SD} \pm 26)$. The MRC dyspnea scores were available for 30 participants. The scores ranged from Grade 1 (not troubled with breathlessness except with strenuous exercise) to Grade 4 (too breathless to leave the house or breathless when dressing or undressing), with $53.3 \%$ of participants reporting dyspnea at a Grade 3 level. Sample characteristics are summarized in Table 1.

Mean scores for MFI subscales ranged from 11.1 (general fatigue) to 13.5 (physical fatigue), as seen in Table 2. Reliability (Cronbach's $\alpha$ ) scores for the subscales were: general fatigue 0.63 ; physical fatigue 0.69 ; reduced activity 0.67 ; reduced motivation 0.70 ; and mental fatigue 0.76 . No associations were detected between fatigue subscale scores and gender, age group, sleep quality, 6MWT distance, MRC dyspnea grade, use of supplemental oxygen, high or low oxygen saturation levels, smoking status, or presence of three or more comorbidities.
Almost half (42.9\%) of the participants had experienced anxiety over the previous week. Scores on the HADS classified nine $(21.4 \%)$ participants as experiencing mild anxiety during the previous week, eight $(19.0 \%)$ with moderate anxiety, and one $(2.4 \%)$ with severe anxiety. Most participants (78.6\%) had no symptoms of depression, although eight (19.0\%) had mild symptoms and one $(2.4 \%)$ experienced severe symptoms of depression. The participant who was classified as experiencing severe symptoms of anxiety also had mild symptoms of depression, while the individual classified as having severe symptoms of depression had a moderate score on anxiety.

The majority of participants $(69.0 \%)$ were considered to experience poor sleep quality. Eight participants (19.0\%) rated the quality of their sleep as fairly or very bad. Participants' mean duration of sleep was 6.9 hours per night $(\mathrm{SD}=1.4)$, although the mean number of hours spent in bed was $8.5(\mathrm{SD}=1.2)$. One-third $(33.0 \%)$ of participants reported that they awoke once a week or more because they could not breathe comfortably, with an equal proportion reporting that they awoke once a week or more because of pain. Medications to promote sleep were used by 14 (33.3\%) participants once a week or more.

Table 3 presents the results of correlational analyses between fatigue, anxiety and depression, sleep quality, and demographic and clinical variables. Significant positive correlations were noted between age and reduced activity $(\rho=0.43, P<0.01)$, and age and reduced motivation ( $\rho=0.31, P<0.05)$. An inverse relationship was found between anxiety and reduced motivation $(\rho=-0.47$, $P<0.01)$.

\section{Discussion}

In contrast with the $43 \%-58 \%$ prevalence of fatigue reported by others for persons with COPD in general, ${ }^{4,5,21}$ fatigue was experienced by almost all participants in this sample of pulmonary rehabilitation patients. Physical fatigue and fatigue related to reduced activity were the MFI subscales with the highest (most negative) scores at 13.5 and 13.0, respectively. Our findings are similar to those of Breslin et al, ${ }^{28}$ who also found that physical fatigue (14.5) and reduced activity (14.2) were dimensions of the MFI most negatively rated by persons with COPD. The mean subscale score for reduced motivation (12.8) in our sample was higher (worse) than scores reported by Hagelin et al for patients in palliative care (11.1), patients receiving radiotherapy (9.9), outpatients (8.3), or hospital staff (7.0), ${ }^{27}$ and by Breslin et al for persons with COPD (11.1). ${ }^{28}$ While neither our study nor that of Breslin et al found 
Table I Participants' demographic characteristics and health status

\begin{tabular}{|c|c|c|c|c|c|}
\hline Characteristics & $n$ & $\mathbf{M}$ & SD & Frequency & Percentage \\
\hline Age (years) & 42 & 72.9 & \pm 6.5 & & \\
\hline Sex & 42 & & & & \\
\hline Male & & & & 17 & 40.5 \\
\hline Female & & & & 25 & 59.5 \\
\hline Pulmonary function tests & 14 & & & & \\
\hline $\mathrm{FEV}_{1}$ (\% predicted) & & 47.8 & \pm 16.3 & & \\
\hline FVC (\% predicted) & & 78.9 & \pm 18.1 & & \\
\hline $\mathrm{FEV}_{\mathrm{I}} / \mathrm{FVC}$ & & 0.68 & \pm 0.13 & & \\
\hline Smoking status & 42 & & & & \\
\hline Exsmokers & & & & 33 & 78.6 \\
\hline Current smokers & & & & 7 & 16.7 \\
\hline Nonsmokers & & & & 2 & 4.8 \\
\hline MRC dyspnea scale & 30 & 2.6 & & & \\
\hline Comorbidities & 42 & 2.6 & \pm 1.4 & & \\
\hline $\begin{array}{l}\text { Amount of pulmonary rehabilitation } \\
\text { received (months) }\end{array}$ & 42 & 25 & \pm 26 & & \\
\hline 6MWT (meters) & 41 & 356 & \pm 90 & & \\
\hline Oxygen saturation & 42 & $92.6 \%$ & \pm 4.1 & & \\
\hline Heart rate (beats/min) & 42 & 102 & \pm 14.4 & & \\
\hline Supplemental oxygen use & 42 & & & & \\
\hline Yes & & & & 11 & 26.2 \\
\hline No & & & & 31 & 73.8 \\
\hline
\end{tabular}

Abbreviations: $n$, number of participants; M, mean; SD, standard deviation; MRC, Medicial Research Council; FEV,/FVC, forced expiratory volume in one second/forced vital capacity; 6MWT, 6-Minute Walk Test.

associations between the fatigue dimension of reduced motivation and depression, ${ }^{28}$ this particular dimension of fatigue may need additional exploration in persons with COPD.

Anxiety and depression are well recognized to be common comorbidities in persons with COPD and often occur together.$^{36}$ Furthermore, anxiety and depression have been found to contribute significantly to variance in the functional status for people with COPD. ${ }^{37}$ In our study, symptoms of anxiety were reported by almost half of participants, with $21.4 \%$ categorized in the moderate to severe anxiety range by the HADS. Similar results were reported by Blinderman et al in a population of persons with severe COPD. ${ }^{38}$ Our sample may have had a higher prevalence of anxiety as a result of measurement differences and sampling bias due to convenience sampling. There may also be circumstances unique to the pulmonary rehabilitation setting that increase the likelihood of anxiety. Other studies have documented prevalence

Table 2 Mean and standard deviation for the MFI-20 subscales

\begin{tabular}{ll}
\hline Subscale & Mean (SD) \\
\hline General fatigue & $\mathrm{II} . \mathrm{I}(2.3)$ \\
Physical fatigue & $\mathrm{I} 3.5(2.2)$ \\
Reduced activity & $\mathrm{I} 3.0(2.3)$ \\
Reduced motivation & $\mathrm{I} 2.8(2.6)$ \\
Mental fatigue & $\mathrm{II} .2(\mathrm{I} .8)$ \\
\hline Abbreviations: SD, standard deviation; MFI-20, Multidimensional Fatigue Inventory.
\end{tabular}

rates of anxiety in persons with stable COPD in the 10\%-19\% range, ${ }^{36}$ and a $32 \%$ prevalence of moderate to severe anxiety in community-dwelling older adults with COPD. ${ }^{6}$ The inverse relationship between anxiety and the reduced motivation dimension of fatigue is quite interesting. We speculate that the psychologic energy expended in coping with high levels of anxiety may reduce motivation to start activities, although this hypothesis requires empiric testing.

Almost one-quarter of participants in this sample reported symptoms of depression. Our finding is similar to that of several other studies, which found that the prevalence of patients with severe COPD who experienced depression was between $18.8 \%$ and $25.0 \%,{ }^{39,40}$ although this rate is lower than the $56 \%$ reported for older adults with COPD by Walke et al. ${ }^{6}$ While previous studies have reported associations between fatigue, anxiety, and depression, we were not able to detect an association. This may be the result of inability of the HADS to adequately assess depression in this population. ${ }^{36}$ Another reason for the lack of association may be the study sample size, which may have had insufficient power to find an association.

Poor sleep quality was experienced by the majority of participants, although we found no associations between the dimensions of fatigue and sleep quality. While fatigue was a prominent symptom in our sample, a relatively low 
Table 3 Correlations between fatigue dimensions and demographic, clinical, Pittsburgh Sleep Quality Index, and HADS scores

\begin{tabular}{llllll}
\hline & General fatigue & Physical fatigue & Reduced activity & Reduced motivation & Mental fatigue \\
\hline Age & -0.01 & 0.11 & $0.43^{*}$ & $0.37^{*}$ & -0.09 \\
MRC score & -0.11 & -0.33 & 0.12 & -0.09 & -0.04 \\
6MWT & -0.17 & -0.11 & -0.25 & -0.07 & 0.11 \\
Heart rate & 0.02 & -0.21 & -0.08 & 0.09 & -0.04 \\
Oxygen saturation & -0.10 & 0.24 & -0.11 & -0.02 & 0.05 \\
Comorbidities & 0.20 & 0.17 & 0.16 & 0.12 & 0.05 \\
Amount of pulmonary & -0.02 & 0.06 & 0.08 & 0.22 & -0.03 \\
rehabilitation received & & & & 0.05 \\
PSQI (sleep quality) & 0.04 & -0.19 & -0.07 & -0.05 \\
HADS (anxiety) & 0.23 & -0.23 & -0.28 & $-0.47^{* *}$ & -0.22 \\
HADS (depression) & 0.00 & -0.22 & -0.29 & -0.20 & -0.30 \\
\hline
\end{tabular}

Notes: *Correlation is significant at the 0.05 level (two-tailed); **Correlation is significant at the 0.01 level (two-tailed).

Abbreviations: HADS, Hospital Anxiety and Depression Scale; 6MWT, 6-Minute Walk Test; MRC, Medical Research Council; PSQI, Pittsburgh Sleep Quality Index.

proportion (19\%) of participants self-rated their sleep as fairly or very bad, suggesting that the proposed link between fatigue, self-rated sleep quality, and objective sleep quality requires further investigation.

There was a significant positive relationship between age and reduced activity, as well as age and reduced motivation. A possible explanation for this finding is that with the natural process of aging, participants experienced a decrease in physical strength which may have negatively impacted their ability to carry out activities of daily living. With a decrease in physical strength, participants may feel less motivated to engage in activities.

In our study, the dimensions of fatigue were not associated with any of the clinical variables, including dyspnea. This may have resulted from the use of existing health record data, which had been collected at varying times over the course of the program, and from not having the participants' dyspnea and fatigue measured concurrently. Previous studies have noted significant relationships between dyspnea and fatigue. ${ }^{4,9,15,41}$ There have been inconsistent findings on the relationship between activity limitation and fatigue. ${ }^{25,26,42,43}$ However, we speculate that the various assessment time intervals at which the 6MWT was conducted among the participants, and the use of the most recent 6MWT distance available, may account for the lack of a significant relationship between activity limitation and fatigue.

Our study is one of the few to examine different dimensions of fatigue in persons attending pulmonary rehabilitation. The small sample size, convenience sampling, absence of a control group, and low reliability coefficients of the MFI are limitations to our study. Only individuals with COPD who attended pulmonary rehabilitation were included in the study. It is possible that the individuals with COPD who attended pul- monary rehabilitation were more motivated or experienced less anxiety and depression than those individuals with COPD who did not attend pulmonary rehabilitation. Selection bias may have also affected our findings because individuals for whom fatigue was a problem may have been more likely to volunteer for this study. However, the high response rate of $95 \%$ for those who were approached makes this unlikely. Due to the high variability in the amount of pulmonary rehabilitation the participants had received, it is possible that the participants enrolled in the program for a longer period of time had experienced less fatigue. There were also limited data available for dyspnea scores and pulmonary function tests. It would have been beneficial to conduct repeat clinical assessments (pulmonary function tests, oxygen saturation, respiratory rate, MRC scores, and 6MWT) on the same day as questionnaire completion to ensure currency. Longitudinal assessments of fatigue would be useful outcome data for the program. In addition, qualitative data relating to the participants' perceptions of fatigue, its impact on their daily functioning, and their attributions of the causes of fatigue would be helpful in providing a more in-depth portrait of this phenomenon. The reliability coefficients for general fatigue, physical fatigue, and reduced activity subscales of the MFI were less than 0.7 in this sample. The MFI has not been used extensively in the population with COPD, and further testing of the instrument is warranted.

\section{Conclusion}

Fatigue was experienced by almost all participants of a pulmonary rehabilitation program in our study, particularly in terms of physical fatigue and reduced motivation. Scores on the reduced motivation subscale of the MFI for our participants exceeded those previously reported for both COPD and cancer patients, and warrant additional exploration in 
the pulmonary rehabilitation population. Similarly, our finding that rates of anxiety among pulmonary rehabilitation participants appear higher than those reported for the overall COPD population suggests that additional focus may need to be placed on anxiety management strategies within such programs. The incorporation of baseline and ongoing assessments of fatigue in the care of participants of pulmonary rehabilitation programs may provide important data regarding the efficacy of symptom control and rehabilitation strategies.

\section{Acknowledgments}

This study is based on a Master's thesis prepared at the University of Saskatchewan, Saskatoon, Saskatchewan, Canada. This study was supported through a grant from the Lung Association of Saskatchewan to CW. The authors express appreciation to the participants for volunteering their time, and to the Saskatoon Health Region pulmonary rehabilitation staff for assisting with recruiting participants and gathering data.

\section{Disclosure}

The authors report no conflicts of interest in this work.

\section{References}

1. O’Donnell DE, Hernandez P, Kaplan A, et al. Canadian Thoracic Society recommendations for management of chronic obstructive pulmonary disease - 2008 update - highlights for primary care. Can Respir J. 2008;15 (Suppl B):1A-8A.

2. Pawlikowska T, Chalder T, Hirsch SR, Wallace P, Wright DJM, Wessely SC. Population-based study of fatigue and psychological distress. BMJ. 1994;308:763-766.

3. Cullen W, Kearney Y, Bury G. Prevalence of fatigue in general practice. Irish J Med Sci. 2000;171:10-12.

4. Kinsman RA, Fernandez E, Schocket M, Dirks JF, Covino NA. Multidimensional analysis of the symptoms of chronic bronchitis and emphysema. J Behav Med. 1983;6:339-357.

5. Kinsman RA, Yaroush RA, Fernandez E, Dirks JE, Schocket M, Fukuhara J. Symptoms and experiences in chronic bronchitis and emphysema. Chest. 1983;83:755-761.

6. Walke LM, Byers AL, Tinetti ME, Dubin JA, McCorkle R, Fried TR. Range and severity of symptoms over time among older adults with chronic obstructive pulmonary disease and heart failure. Arch Intern Med. 2007;167:2503-2508.

7. Gift AG, Shepard CE. Fatigue and other symptoms in patients with chronic obstructive pulmonary disease: Do women and men differ? J Obstet Gynecol Neonatal Nurs. 1999;28:201-208.

8. Graydon JE, Ross E. Influence of symptoms, lung function, mood, and social support on level of functioning of patients with COPD. Res Nurs Health. 1995;18:525-533.

9. Reishtein JL. Relationships between symptoms and functional performance in COPD. Res Nurs Health. 2005;28:39-47.

10. Oga T, Nishimura K, Tsukino M, et al. Longitudinal changes in health status using the chronic respiratory diseases questionnaire and pulmonary function in patients with stable chronic obstructive pulmonary disease. Qual Life Res. 2004;13:1109-1116.
11. Cramer JA, Spilker B. Quality of Life and Pharmacoeconomics: An Introduction. Toronto: Lippincott-Raven; 1998.

12. Small S, Lamb M. Fatigue in chronic illness: The experience of individuals with chronic obstructive pulmonary disease and with asthma. J Adv Nurs. 1999;30:469-478.

13. Ream E, Richardson A. Fatigue in patients with cancer and chronic obstructive airways disease: A phenomenological enquiry. Int $J$ Nurs Stud. 1997;34:44-53.

14. Goldman SE, Ancoli-Israel A, Boudreau R, et al. Sleep problems and associated daytime fatigue in community-dwelling older individuals. J Gerontol A Biol Sci Med Sci. 2008;63:1069-1075.

15. Theander K, Jakobsson P, Jorgenson N, Unosson M. Effects of pulmonary rehabilitation on fatigue, functional status and health perceptions in patients with chronic obstructive pulmonary disease: A randomized controlled trial. Clin Rehabil. 2009;23:125-136.

16. Piper BF. Fatigue. In: Carrieri-Kohlman V, Lindsey AM, West CM, editors. Pathophysiological Phenomena in Nursing. 3rd ed. St. Louis, MO: Saunders; 2003.

17. Lin JM, Brimmer DJ, Maloney EM, Nyarko E, BeLue R, Reeves WC. Further validation of the Multidimensional Fatigue Inventory in a US adult population sample. Popul Health Metr. 2009;7(18). Available from: http://www.pophealthmetrics.com/content/7/1/18. Accessed 2010 May 10.

18. Moorey S, Greer S, Watson M, et al. The factor structure and factor stability of the Hospital Anxiety and Depression Scale in patients with cancer. Br J Psychiatry. 1991;158:255-259.

19. Cathébras PJ, Robbins JM, Kirmayer LJ, Hayton BC. Fatigue in primary care: Prevalence, psychiatric comorbidity, illness behavior, and outcome. J Gen Intern Med. 1992;7:276-286.

20. Nijrolder E, van der Windt DAWM, van der Horst HE. Prognosis of fatigue and functioning in primary care: A 1-year follow-up study. Ann Fam Med. 2008;6:519-527.

21. Foley D, Ancoli-Israel S, Britz P, Walsh J. Sleep disturbances and chronic illness in older adults: Results of the 2003 National Sleep Foundation Sleep in America Survey. J Psychosom Res. 2004;56:497-502.

22. Kapella MC, Larson JL, Patel MK, Covey MK, Berry JK. Subjective fatigue, influencing variables and consequences in chronic obstructive pulmonary disease. Nurs Res. 2006;55:10-17.

23. Ries AL, Bauldoff GS, Carlin BW, et al. Pulmonary Rehabilitation: Joint ACCP/AACVPR evidence-based clinical practice guidelines. Chest. 2007;131 (Suppl 5):S4-S42.

24. Benzo R, Flume PA, Turner D, Tempest M. Effect of pulmonary rehabilitation on quality of life with patients with COPD: The use of SF-36 summary scores as outcome measures. J Cardiopulm Rehabil. 2000;20:231-234.

25. Lenz ER, Pugh LC, Milligan R, Gift A, Suppe F. The middle-range theory of unpleasant symptoms: An update. Adv Nurs Sci. 1997; 19:14-27.

26. Smets EMA, Garssen B, Bonke B, de Haes JCJM. The Multidimensional Fatigue Inventory (MFI): Psychometric qualities of an instrument to assess fatigue. J Psychosom Res. 1995;39:315-325.

27. Hagelin CL, Wengstrom Y, Runesdotter S, Furst CJ. The psychometric properties of the Swedish multidimensional fatigue inventory MFI-20 in four different populations. Acta Oncol. 2007;46:97-104.

28. Breslin E, van der Schans C, Breukink S, et al. Perception of fatigue and quality of life in patients with COPD. Chest. 1998;114: 958-964.

29. Breukink SO, Strijbos JH, Koorn M, Koeter GH, Breslin EH, van der Schans CP. Relationship between subjective fatigue and physiological variables in patients with chronic obstructive pulmonary disease. Respir Med. 1998;92:676-682.

30. Zigmond AS, Snaith RP. The Hospital Anxiety and Depression Scale Acta Psychiatr Scand. 1983;67:361-370.

31. Dowson C, Laing R, Barraclough R, et al. The use of the Hospital Anxiety and Depression Scale (HADS) in patients with chronic obstructive pulmonary disease: A pilot study. $N$ Z Med J. 2001;114: $447-449$. 
32. Buysse DJ, Reynolds CF III, Monk TH, Berman SR, Kupfer DJ. The Pittsburgh Sleep Quality Index: A new instrument for psychiatric practice and research. Psychiatry Res. 1989;28:193-213.

33. Carpenter JS, Andrykowski MA. Psychometric evaluation of the Pittsburgh Sleep Quality Index. J Psychosom Res. 1998;45:5-13.

34. Fletcher CM. Standardized questionnaire on respiratory symptoms: A statement prepared and approved by the Medical Research Council's Committee on the Aetiology of Chronic Bronchitis. BMJ. 1960;2:1665.

35. American Thoracic Society. ATS statement: Guidelines for the sixminute walk test. Am J Respir Crit Care Med. 2002;166:111-117.

36. Maurer J, Rebbapragada V, Borson S, et al. Anxiety and depression in COPD: Current understanding, unanswered questions and research needs. Chest. 2008;134 (Suppl 4):S43-S56.

37. Seung HF, Kunik ME, Molinari VA, et al. Functional impairment in COPD patients: The impact of anxiety and depression. Psychosomatics. 2000;41:465-471.
38. Blinderman CD, Homel P, Billings JA, Teenstedt S, Portenoy RK. Symptom distress and quality of life in patients with advanced chronic obstructive pulmonary disease. J Pain Symptom Manage. 2009;38:115-123.

39. Di Marco F, Verga M, Reggente M, et al. Anxiety and depression in COPD patients: The roles of gender and disease severity. Respir Med. 2006;100:1767-1774

40. van Manen JG, Bindels PJE, Dekker FW, IJzermans CJ, van der Zee JS, Schadé E. Risk of depression in patients with chronic obstructive pulmonary disease and its determinants. Thorax. 2002;57:412-416.

41. Janson-Bjerklie S, Carrieri VK, Hudes M. The sensations of pulmonary dyspnea. Nurs Res. 1986;35:154-159.

42. Woo K. A pilot study to examine the relationships of dyspnoea, physical activity and fatigue in patients with chronic obstructive pulmonary disease. J Clin Nurs. 2000;9:526-533.

43. Woo K. Physical activity as a mediator between dyspnea and fatigue in patients with chronic obstructive pulmonary disease. Can J Nurs Res. 2000;32:85-98.

\section{Publish your work in this journal}

The International Journal of COPD is an international, peer-reviewed journal of therapeutics and pharmacology focusing on concise rapid reporting of clinical studies and reviews in COPD. Special focus is given to the pathophysiological processes underlying the disease, intervention programs, patient focused education, and self management protocols.

\section{Dovepress}

This journal is indexed on PubMed Central, MedLine and CAS. The manuscript management system is completely online and includes a very quick and fair peer-review system, which is all easy to use. Visit http://www.dovepress.com/testimonials.php to read real quotes from published authors. 